\title{
Immunologic Factor Interaction
}

National Cancer Institute

\section{Source}

National Cancer Institute. Immunologic Factor Interaction. NCI Thesaurus. Code C40512.

Immunologic Factor Interaction involves temporary non-covalent binding through intermolecular physical forces of attraction and often spatial complementarity with antibody or cytokine proteins that affect immune functions, immune responses, or inflammation. 